\title{
A different look at perceptual defense for taboo words
}

\author{
BRUCE DENNIS SALES AND RALPH NORMAN HABER
}

THE UNIVERSITY OF ROCHESTER

Haber has concluded from previous work that meaningfulness, frequency, and related stimulus variables directly affect the clarity of letters of words. To examine whether the letters of taboo words would be harder to see, 12 taboo words were matched in length to 12 very frequent and 12 very rare neutral words. Each word was shown an unpredictable number of times. Three groups of $8 \mathrm{Ss}$ each were required, respectively, either to name the letters they saw on each flash, name the letters and then guess the word, or guess the word only. While no differences were found among the three groups, the clarity of the letters of the frequent words exceeded that for the rare words, which in turn was higher than that of the taboo words. This difference was apparent on even the first flash, supporting the interpretation of a perceptual defense effect.

In a continuing series of experiments, it has been shown that the perceived clarity of letters in words, permitting the $S$ to see more letters, will increase with well spaced repeated presentations of the words without any changes in intensity or duration. The increase in clarity with repetition follows a negatively accelerated growth curve. The shape of that curve, reflecting the rate of increase in clarity is unaffected by variation in the frequency of words (rare or frequent English words; Haber, 1965), the language of the words (English or Turkish; Hershenson \& Haber, 1965), prior knowledge of the stimulus (whether the subject knows which word is going to be exposed on the next trial or not; Haber, 1965), familiarity of the words (English or nonsense; Haber \& Hershenson, 1965; Hershenson, 1965; Haber \& Meiselman, in preparation), number of letters in the word (one or four or seven; Dainoff \& Haber, 1966; Haber \& Hillman, 1966; Haber \& Meiselman, in preparation; Haber, 1967), and the duration at which the words are exposed (Haber \& Hershenson, 1965; Haber, 1967).

While the rate of increase in perceptual clarity is invariant over these stimulus and subject conditions, the amount of perceptual clarity is not. Thus, it is easier to see the letters of English words as compared to Turkish words, even though the rate of increase in clarity is the same. The curve of English words is above but parallel to that of the Turkish words. Perceived clarity is higher for frequent as compared to rare words, for English as compared to Turkish words, for English as compared to nonsense words, for words given prior knowledge of what word is being flashed as compared to no prior knowledge, for long as compared to short words, and for words presented at high as compared to low durations.

In summary then, the perceived clarity of letters of words is affected by a wide range of stimulus variables, even though those variables have no effect on the rate of increase in perceived clarity with repetition. Haber has concluded (1967) that each of these variables can affect the perceptual clarity of the stimulus directly, and that these effects are not being mediated by response mechanisms such as response bias or response inhibition. In order to investigate this generalization further, the present experiment was designed with taboo and neutral words, in the hope that a more direct answer could be given as to whether the emotional meaning of stimuli can affect perceived clarity.

Several lines of evidence are available from the previous experiments to support the assumption that perceived clarity, and not just response bias processes, is being manipulated. A number of operations were used to control or remove the possibility that the $S$ was in fact guessing unseen letters based upon information that he gained from the letters he had seen, rather than just reporting the letters that he could see. To support this conclusion, the following points have been demonstrated: (1) Nearly every S in all of the experiments spontaneously volunteered the information that he thought $\mathrm{E}$ had been increasing the duration or the intensity of the stimulus from flash to flash. Thus, the phenomenal impression that each $S$ gained about the stimulus from repetition alone seemed to be equivalent to what he would see if the energy had been increased. (2) The increase in clarity of the letters of the word was not always perfect. There were a number of occasions when each $S$ would report on a particular trial seeing a certain letter clearly and then indicate on the next trial not seeing that letter. Such a contradictory response could only be the result of a report of his perception rather than of his knowledge about what he thought the stimulus was. (3) In the experiment testing prior knowledge (Haber, 1965), it was found that the rate of increase in clarity as a function of repetition was identical whether the $\mathrm{S}$ was shown just prior to each flash of the word what the word was going to be as compared to when he had no prior knowledge of the stimulus. With complete prior knowledge, there should be no variance attributed to guessing since the $\mathrm{S}$ knows exactly what the stimulus will be. The fact that the same rate of increase in clarity as a 
function of repetition was found suggests that that variance must be due to something other than guessing. (4) To control the possibility that the $\mathrm{S}$ switches his fixation from flash to flash of a word and hence figures out what the first part of a word is and then the middle then the end, for example, Haber and Hillman (1966) showed that clarity would increase with repetition for a single letter subtending only $20 \mathrm{~min}$ of visual angle, a stimulus for which changes in fixation would be irrelevant. (5) To examine the possibility that the subject was not reporting an increase in clarity but rather an increase in willingness to make a response without any change in his sensitivity to the stimulus, Haber and Meiselman (in preparation) constructed stimuli with missing or deformed letters in them so that an assessment could be made of the S's false alarm rate as well as his hit rate of correctly naming lettèrs. We found very clear evidence that while the S's ability to identify correctly drawn letters (hit rate) increased with repetition, the false alarm rate held level or decreased with repetition. If these two parameters are examined together according to signal detection theory, it would be clear that the S's sensitivity to the stimulus is increasing with repetition and that this increase cannot be attributed to a change in his criteria.

With these data at hand it seems reasonable to examine in this context the perceptual defense paradigm, in which a comparison can be made between the clarity of letters of emotionally neutral versus taboo words. Will the rate of increase in clarity with repetition be invariant over variation in the emotional content of the stimulus? Further, will it be easier to see letters of neutral words, as compared to taboo words? If the same rate of increase in clarity for taboo word $\$$ is found as for neutral words, but with an absolute difference in amount of clarity, then it. could be concluded that the emotional content of the stimulus was affecting the clarity of the percept directly.

Two controls were added, one for the frequency of the words and one for response suppression. Since it is not possible to estimate the frequency of taboo words as compared to neutral words, nor even whether the appropriate control is for spoken frequency or written frequency, two groups of neutral words were selected whose frequencies were known either to be extremely high or extremely low. It was assumed that the taboo word frequencies both for written and spoken usage were probably between these two extreme groups of words.

As a way of examining effects of response suppression, three groups of Ss were used differing in the response required. One group was asked to report on each flash the letters that they could see, a second to report the letters that they could see and then guess what the word might be, and a third only to guess what the word might be. This last group is the one most equivalent to the typical experiments on word recognition, in which the Ss are instructed to use a guessing indicator. The $S s$ in the first group presumably should show the least response suppression because they are not required to name an emotionally laden response which might produce some embarrassment in an interpersonal situation. The middle group was run in order to provide a control to compare the first and the third group.

\section{Subjects}

\section{METHOD}

Three groups of eight male undergraduates enrolled in the large Introductory Psychology course at the University of Rochester served as Ss. They were tested individually in two sessions at least $24 \mathrm{~h}$ apart. They were all naive as to the purpose of the experiment. Five additional Ss had been run and dropped from the experiment when they admitted in an extensive post-experimental interview that they had been aware of and thought about the purpose of the experiment during its course.

Each $S$ was assigned a number from 1 to 3 from a table of random numbers which determined the group to which he was assigned. When one of the three groups was filled, that number was eliminated until there were eight Ss in each of the three groups.

\section{Stimuli}

Each $S$ was shown 76 words in each of the two sessions. The first session was designed for adaptation to the experimental procedures and to thoroughly practice the $S$ on the task so there would be no further changes in performance over time. No taboo words were shown in the first session. In the second session, 12 taboo words were shown and to each of these, 12 frequent and 12 rare neutral words were matched. Table 1 lists these 36 words which comprise the stimuli on which all analyses are based. The remaining 40 words in the second session were all seven-letter, three-syllable English words, 20 rare and 20 frequent, randomly drawn from lists used by Haber (1965).

Table 1. List of matched words

\begin{tabular}{llc} 
Taboo & Rare neutral & Frequent neutral \\
\hline fuck & coot & help \\
eunt & pied & same \\
shit & mace & take \\
cock & mull & with \\
prick & bylaw & place \\
bitch & extol & story \\
penis & grime & voice \\
vagina & dotage & office \\
breast & illume & should \\
douche & obtuse & become \\
bastard & aconite & opinion \\
clitoris & barnacle & interest \\
\hline
\end{tabular}


In the first session, the 76 words were very similar except for the absence of taboo words. Another set of 12 frequent words and 12 rare words matched in length from the Thorndike-Lorge counts were included in the first session along with 52 words selected from Haber (1965), half of which were rare and half frequent.

The 12 taboo words were selected by the authors who judged them to be words that would cause an individual anxiety and tension if he had to repeat them in an interpersonal situation. They were matched by number of letters to 24 frequent neutral and 24 rare neutral words, 12 of each of these appearing in the second session. Words were considered rare if they had a frequency of 0 to 10 per million on both the $\mathrm{L}$ and $S$ lists from Thorndike-Lorge (1944). Words were considered frequent if they had a frequency of 1000 or more occurrences on both the $L$ and $S$ lists. The matched words were picked by taking the first one on the first page that met the criteria and then skipping the next five pages and repeating the process. Those words which might have obvious emotional connotations (e.g., buxom, armpit) and those which were capitalized were skipped.

The ordering of the words for each session was randomized, with the restriction that for the second session none of the 36 critical items appeared among the first 15 words. This restriction was imposed to permit $S$ to readapt and remove whatever transient practice effects might occur in the beginning of the second session.

\section{Apparatus}

Stimuli were presented singly in the rear of one channel of a three-channel mirror tachistoscope (Scientific Prototype Model GA). A second background channel contained faint lines above and below the word which served as fixation boundaries. This channel was on at all times except when the stimulus channel was on. Stimulus durations were monitored and varied by less than 1 per cent. The luminance measured at the eye piece with a Gamma Scientific photometer was $10 \mathrm{ft}-\mathrm{L}$ at all times.

The words were printed on white cards $8-3 / 4$ in. $x$ 6-3/4 in. using a LeRoy lettering stencil 33240290CL and pen 3233-1. The letters were spaced evenly in a row $3 / 8$ in. high and 5/16 in. between the center of neighboring letters $(20 \mathrm{~min}$ and $35 \mathrm{~min}$ of visual angle, respectively).

\section{Procedure}

The first session was used to determine each S's threshold, to absorb practice effects, and to diminish the S's expectancy of viewing anything but neutral English words. Each word was assigned to one of seven duration values ranging from 5 to $35 \mathrm{msec}$ in steps of $5 \mathrm{msec}$, and one of six repetition numbers $(1,2,3,4,5$, or 10$)$, representing the number of times each word would be exposed. The interval between repetitions of a word was never less than $8 \mathrm{sec}$. Thus, one word was assigned to at least one of the 42 duration-by-trial combinations. The S's threshold was defined as that stimulus duration for which on the first flash only an occasional letter was perceived while by the fourth or fifth flash most of the letters of the word would be reported. High and low durations were progressively eliminated as the session went on as the threshold became apparent. Since the threshold varied with the size of the word, an average threshold was determined for the four- to eight-letter words.

The second session was conducted at least $24 \mathrm{~h}$ after the first one. The duration of the stimulus presentations was kept constant at the S's threshold as determined in the first session. Six repetition numbers $(1,2,3,4,5$, and 10$)$ were used, so that two taboo words were shown for one flash, two for two flashes, and so forth. These repetition numbers were assigned randomly to all of the words, with words matched being assigned the same number of repetitions.

The instructions were read to each $S$ at the beginning of each session. The three groups received the same instructions except for information about the nature of their reports:

(For all groups) "This is a perception experiment. A list of words will be flashed on the screen one at a time for very short durations. (For the lettersonly group) You are to report those letters which you see on each presentation and the relative positions of the letters in the word. It is not necessary for you to guess what the word is. I am only interested in the letters that you are sure you see on each flash and where they appeared. Begin reporting letters as soon as the word flashes. (For the letters-then-guess group) You are to report those letters which you are sure you see on each presentation and the relative position of the letters in the word. Then try to guess what the word is. Do not try to guess until after you have reported just those letters you are sure you see. I am basically interested in those letters and not in the word. Begin reporting the letters as soon as the word flashes. (For the guess-only group) On each presentation of the word try to guess what it is. Report your guess for the word as soon as it flashes. (For all groups) Some words will only be flashed once while others will be flashed several times. Since you will not know how many times a word will be repeated, treat each trial as if it would be the last presentation of that word. I will always inform you when a new word is to be presented. A word will not be flashed until you press the button next to you. When I give you the signal, make sure you are prepared to view the word, and then press the button. If you have any questions, please ask them now since I will not be able to answer any once the experiment has begun."

At the end of the second session, $E$ discussed the 


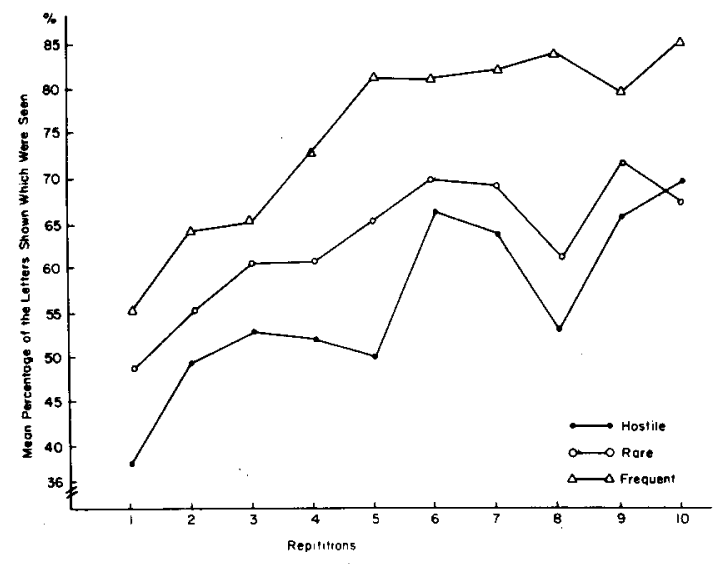

Fig. 1. The mean percentage of letters seen on each flash for frequent, rare, and taboo words as a function of the number of repetitions of each word. Data from two groups combined, $\mathbf{N}=16$.

experiment at some length with each S, particularly making note on any inklings of the nature of the experiment or concern during the course of the experiment as to what it was about. Then the purposes of the experiment were explained to each $\mathrm{S}$.

\section{RESULTS AND DISCUSSION}

The data for the number of letters seen were scored according to two criteria. A S perceived a particular word for a particular flash if for that flash he reported seeing all of the letters of the word. The other criteria was merely to count the number of letters that the $S$ reported seeing on each flash, and express that as a percentage of the total number of letters presented (the percentage correction was necessary since the words had differing numbers of letters). Since for every analysis carried out the two scoring systems yielded identlcal results, all analyses to be reported are based on the second criterion.

The mean percentage of letters reported for each flash for each word for the letters-only group was compared with the comparable score for the lettersthen-guess group. No differences on any of the comparisons were found between these two groups. Likewise, the letters-then-guess group was compared with the guess-only group on their accuracy in guessing the stimulus. For no comparison was there any difference between these two groups. Since the analyses of principal interest concerned that of reporting letters, the letter data from Groups 1 and 2 were combined. All further comparisons will be based upon $16 \mathrm{Ss}$.

Figure 1 presents the results of the increase in percentage of letters seen as a function of repetition for the three groups of words-frequent, neutral, and taboo. An analysis of variance was performed on the data in this table. It showed significant main effects of repetition $(p<.001)$ and type of word $(p<.001)$. There were no significant interactions. Thus, for all words $S$ was able to see more of the letters with more repetitions even though there was no increase in the energy. Second, holding repetition constant, it was easier to see letters of frequent words than rare words (a finding reported in earlier research by Haber, 1965), and further, that the taboo words were the most difficult to see of all. On the assumption that the frequency of the taboo words is in fact higher than the rare neutral words, this difference cannot be accounted for by the frequency effect. Consequently, it must be due to some emotional content of the taboo word which makes it more difficult for $S$ to see the individual letters.

The analysis of variance indicated no interaction between the number of repetitions and the type of words, implying that the leveling of the taboo word growth curve for Trials 3,4 , and 5 is not a significant variation from the gain in the other curves. One should not make too much of this leveling because of the small number of words being represented in this data and the tenuous nature of this break in the curve. However, it could be due to a perceptual effect in that as the $S$ begins to recognize the whole word, the individual letters become even harder to see. It is also possible that a response suppression effect is occurring in that as the $S$ begins to recognize the whole word he suppresses his reports of the letters until he is more certain. Because of the extremely few words in this study it is not possible to disentangle these possibilities. It should be noted though that a perceptual defense effect is evident even on the first flash of the words, in that fewer letters are seen of the taboo words than of the words of the other types.

There is a possibility that the defense effect on the first trial is non-perceptual, but the result of a few Ss having guessed the entire word on the first flash and then suppressing their report of the letters. Presumably these Ss would be ones whose ability to see the letters of any kind of word would be very high. To test this, the 16 Ss were divided at the median, based on their performance on the frequent words. The pattern of results on the rare and taboo words was identical for these two subgroups, with the better Ss on the frequent words also being better on the taboo words. Thus, the defense effect on the first flash cannot reasonably be due to a few good Ss being able to guess the word and then suppressing their letter reports.

The control in this experiment over response suppression was by comparing the different instructions regarding what to report. No differences were found. However, this is not a very elegant control since it is quite possible that an $\mathrm{S}$ would suppress the naming of letters of a taboo word as much as naming the word itself, since he knows the E knows he knows the word from just the letters alone. This experiment was not primarily concerned about response suppression and this null finding is offered only as a weak indication 
that an equal amount of response suppression was present in each condition, and that therefore response suppression cannot account for the perceptual effects noted. The large differences on the initial flash between the taboo and neutral words also suggests that response suppression cannot easily account for these data.

Because of the unfortunate limitation in English of having only a relatively small number of widely known taboo words, it is not possible to increase the reliability of the data by adding more stimuli. However, these results will support several fairly limited conclusions. (1) It is another study which shows that the clarity of individual letters increases with repetition. (2) This effect occurs for taboo words as well as frequent and rare neutral words. (3) It is harder to see the individual letters of the taboo words than either very frequent or very rare neutral words. (4) Response suppression does not seem to be a factor in this difference, at least to the extent that no differences were found between the letters-only, the letters-then-guess, and the guess-only groups. This suggests that the emotionality of the taboo words interferes with the clarity of their percepts. This suggests then a direct effect of emotionality on perception (a perceptual defense effect).

\section{References}

Dainoff, M., \& Haber, R. N. How much help does repetition give to recognition processes? Percept. \& Psychophys., 1967, 2, 131136.

Haber, R. N. The effect of prior knowledge of the stimulus on word recognition processes. $J$. exp. Psychol., 1965, 69, 282-286.

Haber, R. N. Repetition as a determinant of perceptual recognition processes. In J. C. Mott-Smith, W. Wather-Dunn, H. Blum \& P. Lieberman (Eds.), Symposium on models for the perception of speech and visual form. Cambridge: MIT Press, 1967. Pp. 202212.

Haber, R. N., \& Hershenson, M. The effects of repeated brief exposures on the growth of a percept. J. exp. Psychol., 1965, $69,40-46$.

Haber, R. N., \& Hillman, E. R. Changes in single letter clarity with repetition. Percept. \& Psychophys., 1966, 1, 347-350.

Haber, R. N., \& Meiselman, C. H. Estimates of flase-alarm reports in studies of perceptual recognition. In preparation.

Hershenson, M. Perceptual microgenesis as a function of "word" structures. Paper read at the Midwest. Psychol. Assoc. Convent., Chicago, Illinois, April, 1965.

Hershenson, M., \& Haber, R. N. The role of meaning in the perception of briefly presented words. Canad. J. Psychol., 1965, $19,42-46$.

\section{Note}

1. This research was supported in part by United States Public Health Research Grant MH 10753 to the second author. We are most indebted to Thomas Natsoulas for some excellent comments on an earlier draft.

(Accepted for publication October 15, 1967.) 